\title{
Preparation and Properties of Rice Husk-based Activated Carbon Using Phosphoric Acid as Activator Xinghui Chen ${ }^{1}$,Ying Fang ${ }^{1}$, Jian Zhu', Fuyong Wang ${ }^{1}$, Teng Zhao ${ }^{1}$ and Dongli Fan ${ }^{2, a^{*}}$
}

${ }^{1}$ Suzhou institute of supervision \&inspection on product quality,suzhou,jiangsu 215000,China, ${ }^{2}$ Nantong university, College of chemistry and chemical engineering, Nantong, Jiangsu 226000, China

afdlsky@163.com

\section{Keywords: Rice husk; Activated Carbon;Activate; Adsorption;Phosphoric acid}

\begin{abstract}
The rice husk through special processing by phosphoric acid activated and carbonized in one step at a certain temperature. the adsorption of benzene has been measured. The effects of such factors as the ratio of phosphate to rice husks, the activating temperature and time, heating rate and different activation process upon the adsorption properties of active carbon have been investigated. Research shows that, the optimal ratio of phosphate to rice husks was $1: 2$, the optimal activating temperature is $500{ }^{\circ} \mathrm{C}$, and the optimal activating time is 3 hours. To reduce the heating rate appropriately can improve the properties of the adsorption of activated carbon for benzene.
\end{abstract}

\section{Introduction}

It is difficult to avoid the shortage of the activated carbon raw material because of the dwindling of the world's forests and coal resources. Therefore, it has gradually become a hot research to find other raw materials for activated carbon preparation. Rice husk resource is abundant in China, but the rice husk utilization and conversion rate are low and the environmental pollution is serious.Rice husk have high content of fixed carbon and can be used as a good raw material for the preparation of activated carbon[1-2].

In the modem activated carbon industry, advocating environment protection and energy saving, phosphoric acid activated has been paid more attention with low energy, simple operation and less pollution[3-4].In this experiment,active carbon was prepared from rice husk, which was treated with phosphoric acid. The effects of such factors as the ratio of phosphate to rice husks, the activating temperature and time, heating rate and different activation process upon the adsorption properties of active carbon were investigated.

\section{Experimental}

Materials. Rice husk was obtained from a rice mill in Nantong, China, in the autumn of 2014.Rice husk was initially washed thoroughly with distilled water to remove any impurities, such as adhering soil,dust and parts of metal.After drying at $120^{\circ} \mathrm{C}$ for $10 \mathrm{~h}$,these precursors were finely ground into 30 mesh.The finer fraction was used for characterization and for the activation studies. All chemicals and solvents used in this study were analytical grade and used without further purification.Phosphoric acid and ethanol were provided by Tianjin Kermel Co.Distilled water was applied for all synthesis and treatment processes.

Production of Activated Carbon by H3PO4 Activation. Rice husk was mixed with 85wt\% phosphoric acid, the impregnation ratio of phosphoric acid were from $1: 1$ to $3: 1$. The activation reactions were carried out at the high temperature ranging from $500^{\circ} \mathrm{C}$ to $700^{\circ} \mathrm{C}$ with the heating rate $600^{\circ} \mathrm{C} / \mathrm{h} 、 300^{\circ} \mathrm{C} / \mathrm{h} 、 150^{\circ} \mathrm{C} / \mathrm{h}$ and maintained for $2-4 \mathrm{~h}$. The product was thoroughly washed with 
hot distilled water to remove the excess phosphorus compounds until the wash water attained $\mathrm{pH} 7$. The washed activated carbon samples were dried at $110{ }^{\circ} \mathrm{C}$ for $12 \mathrm{~h}$, ready for adsorption test.

Adoption. Equilibrium sorption studies were conducted in a set of pre-dried empty weighing bottles containing adsorbent.These bottles were dried in a vacuum oven for $2 \mathrm{~h}$ and put in benzene steam cylinder to adsorb benzene steam until the equilibrium was reached.

Benzene adsorption value $=(\mathrm{m} 3-\mathrm{m} 2) /(\mathrm{m} 2-\mathrm{m} 1)$

Where $\mathrm{m} 1$ is the weight of empty weighing bottle after drying, $\mathrm{m} 2$ is the whole weight of bottle and activated carbon before adsorption, $\mathrm{m} 3$ is the whole weight of bottle and activated carbon after saturated adsorption.

\section{Results and Discussion}

Effect of Impregnation Ratio. The effect of impregnation ratio was studied using heat treatment at $500^{\circ} \mathrm{C}$ for $3 \mathrm{~h}$ at five different impregnation ratios ranging from $1: 1$ to $1: 3$. The product was washed with distilled water to remove residue phosphoric acid and measured the benzene adsorption.The results were shown in Fig. 1.

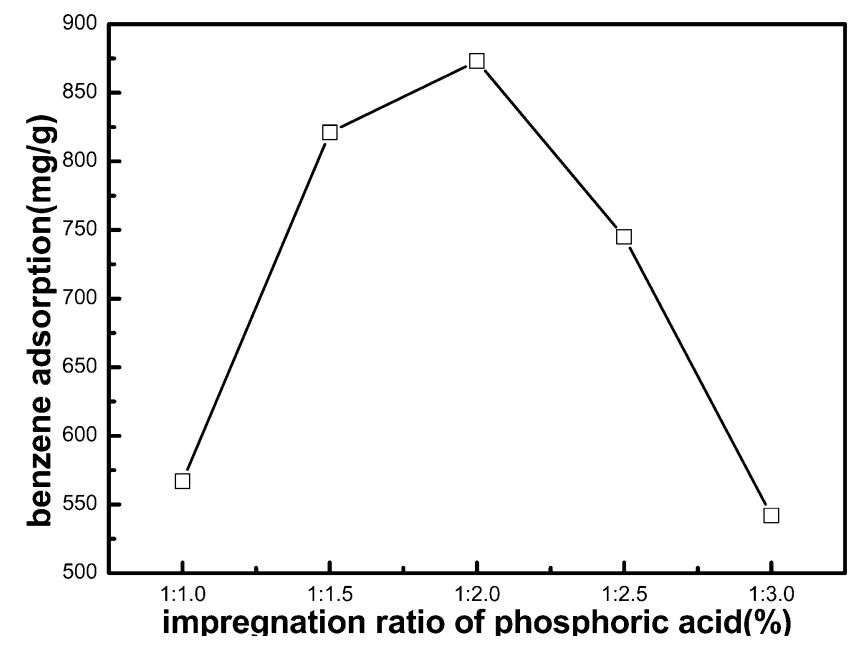

Figure 1.Effect of impregnation ratio of phosphoric acid on the benzene adsorption ability of activated carbons

Fig. 1 shows the variation of benzene adsorption at different impregnation ratios. The results showed that the yield of benzene adsorption increased with the increase in impregnation ratio initially, reached maximum at 1:2 and then decreased. The pores with small specific surface area became small and less because of the incomplete reaction between phosphoric acid and the rice husk caused by less phosphate.

When the phosphate content was too high, on the one hand,the burn-off of activated carbon was too high because of the excessive reaction with carbon.On the other hand, too much residual phosphate blocked pores on the rice surface and prevented the further volatilization, leading to the reduction of porosity and the increasing of specific surface area[5].

Effect of Activation Temperature. Under nitrogen protection, the rice husk with impregnation ratio $1: 2$ which treated by phosphoric acid was put in oven at $400{ }^{\circ} \mathrm{C}$. The activation temperature rose from $200{ }^{\circ} \mathrm{C}$ to $500{ }^{\circ} \mathrm{C}$ with different heating rate and maintain $500{ }^{\circ} \mathrm{C}$ for 3 hours. Then the rice husk was took out directly and the residual phosphate was washed with distilled water after carbonization and activation for 3 hours. And then a series of experiments with the same process but in different activation temperature of $500{ }^{\circ} \mathrm{C}, 600{ }^{\circ} \mathrm{C}, 700{ }^{\circ} \mathrm{C}$ were done. The benzene adoption was measured and the results were shown in Fig. 2. 
If the rice husk was put in oven at room temperature and reach the activation temperature in $1 \mathrm{~h}$, then took out after $3 \mathrm{~h}$ heating and cooling below $200^{\circ} \mathrm{C}$, the results were shown in Fig. 3.

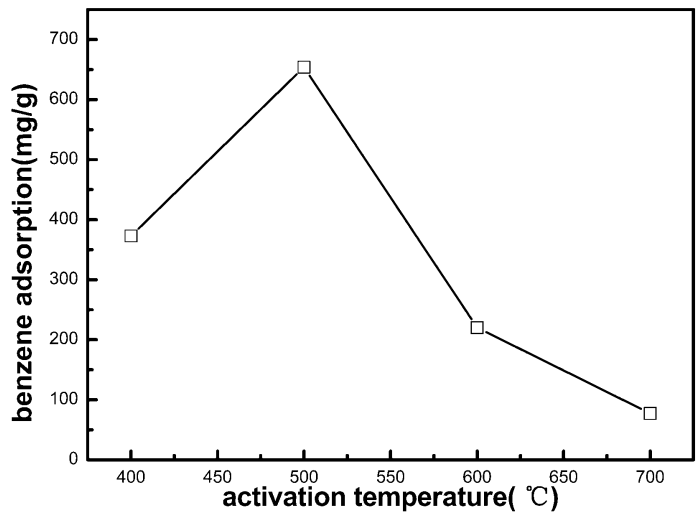

a. $400^{\circ} \mathrm{C}$

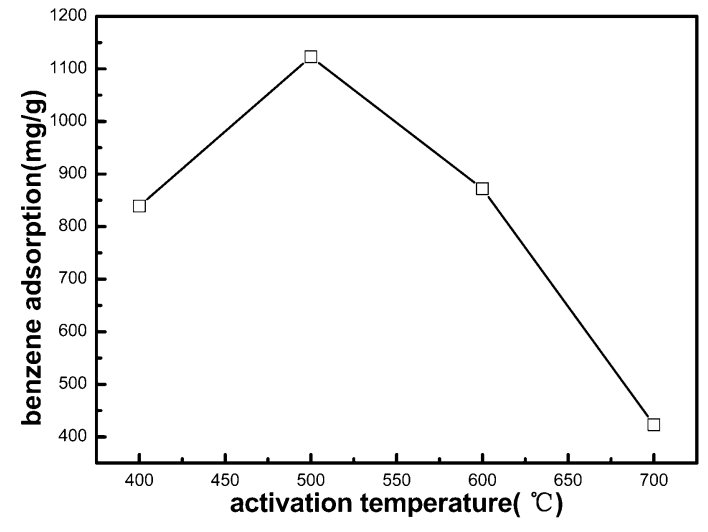

b.room temperature

Figure 2.Effect of activation temperature on the benzene adsorption ability of activated carbons

Fig. 2 depicts the influence of activation temperature $\left(400-700{ }^{\circ} \mathrm{C}\right)$ on the value of benzene adsorption. When the activation temperature ranged from $400{ }^{\circ} \mathrm{C}$ to $700{ }^{\circ} \mathrm{C}$, the benzene adsorption reached the maximum value at $500^{\circ} \mathrm{C}$. The reason is that mesopores were often existed in the activated carbon using phosphoric acid as activator. When the temperature was too low, the pores with small specific surface area became small and less because of the insufficent pyrolysis of rice husk. With the temperature increased above $500^{\circ} \mathrm{C}$, the pore volume decreased. Since progressive heat treatment temperature increased the carbon "burn off", it will result in too big pore[5,6]. These results suggested that to obtain the optimal value of the yield and the pore volume, the temperature should be around $500{ }^{\circ} \mathrm{C}$.

By comparing the two process under different temperature, we found that the benzene adsorption ability of activated carbons in Fig. 2(b) was generally higher than the Fig. 2(a). It may be because rice husk can withstand higher temperature of carbonization and activation temperature after the preliminary low-temperature carbonization process caused by the heating process from low to high temperature. It may also because activated carbon in Fig. 2(b) is took out when cooled to a certain temperature, so the actual activation time is longer. While in Fig. 2(a) processing, the activated carbon is taken out directly under the activation temperature, more rice husk was burned into ash and the specific surface area decreased because the rice husk contacted with oxygen in the air at high temperature which resulting in the further burning of activated carbon[7].

Effect of Activation Time. Five treated rice husk with the impregnation ratio1:2 were put into a box-type resistance furnace and heated to $500{ }^{\circ} \mathrm{C}$ in 1 hour,the temperature insulated respectively for 2.0, 2.5, 3.0, 3.5and $4.0 \mathrm{~h}$. Then the rice husk was took out directly and its benzene adoption was measured after washing the residual phosphate with distilled water,the results were shown in Fig.3. 


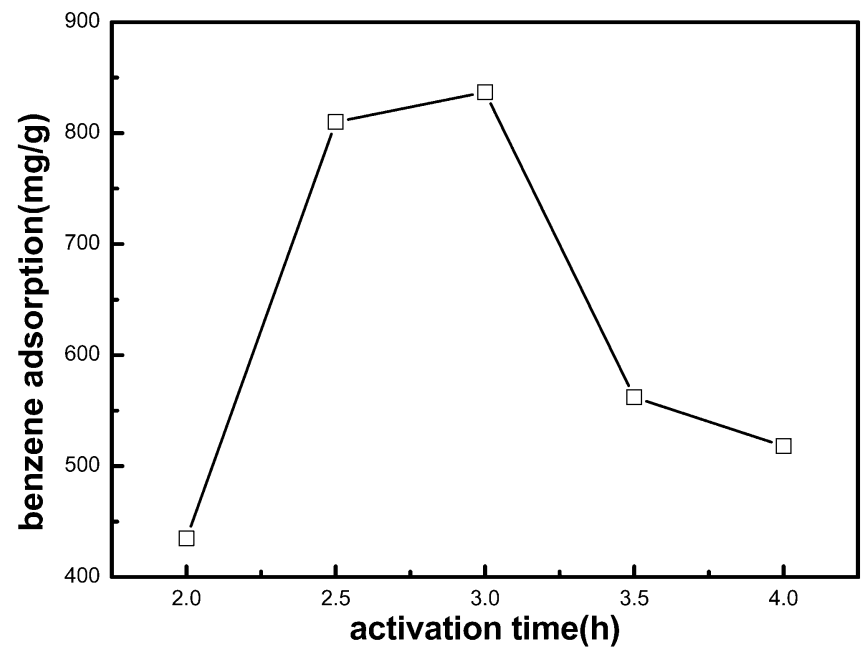

Figure 3. Effect of activation time on the benzene adsorption ability of activated carbons

The Fig. 3 shows that the activated carbon has the best adsorption performance after activating for 3 hours. Whether to reduce or extend the activation time, it is not conducive to improving the adsorption performance of activated carbon. If the activation time is too short, the pyrolysis reaction is not complete and the volatile components cannot be completely evaporate, which resulting in the decreased specific surface area and pore volumes of activated carbons. If the activation time is too long, the reaction can be carried out adequately, the pore volumn increased while the specific surface area of activated carbon is also decreased[8]. At the same time, too long reacted time will cause too high LOI of carbon and more ash content, and the capacity decreased.

Effect of Heating Rate. Under nitrogen protection, the rice husk treated by phosphoric acid was put in oven at $200{ }^{\circ} \mathrm{C}$. The temperature rose from $200{ }^{\circ} \mathrm{C}$ to $500{ }^{\circ} \mathrm{C}$ with different heating rate and maintain $500{ }^{\circ} \mathrm{C}$ for 3 hours. Then the rice husk was took out directly and its benzene adoption was measured after washing the residual phosphate with distilled water,the results were shown in Fig. 4.

As seen in Fig. 4, the adsorption of activated carbon increased with the decrease of heating rate.

This may be because the lower the heating rate, the more completely carbonized activation reaction. The volatiles and reactive gas of rice husk run over slowly with the slowly raising temperature, and was more conducive to the formation of the initial gap which will benefit the further development of space[9,10]. In addition, the lower the heating rate and the longer carbonization time made the formation of porous structure easier.But considering the activation time of activated carbon, the extended reaction time caused by the especially slow heating rate may lead to the adsorption performance degradation. And longer heating time will lengthen production cycle time and further increase the cost,so the process is not suitable for industrial manufacture. 


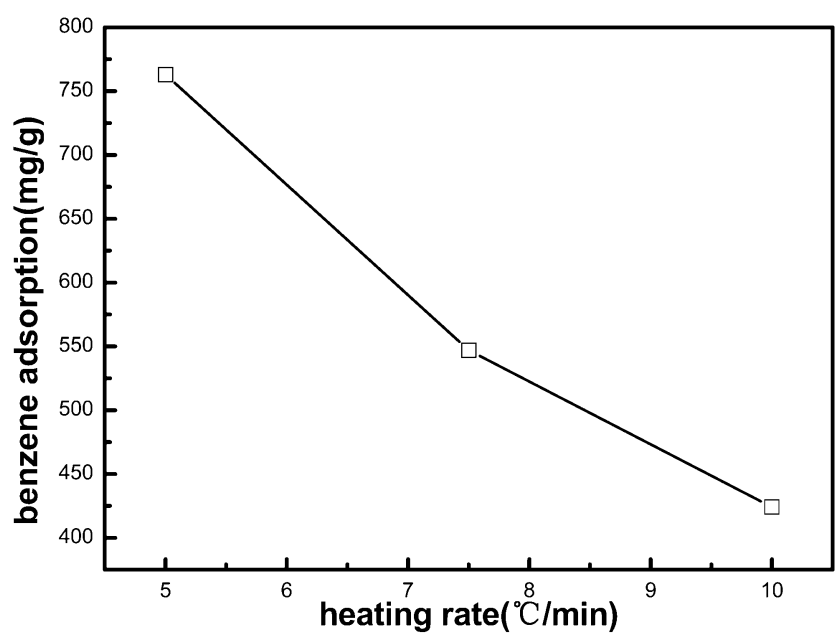

Figure 4. Effect of heating rate on the benzene adsorption ability of activated carbons

\section{Conclusion}

The results obtained showed that rice husk-based activated carbon using phosphoric acid as activator can be readily used for the adsorption of benzene. The carbonization and activation step completed at the same time, the process is simple and easy to industrialization.The adsorption of activated carbon can be controlled by modification of the impregnation ratio of phosphoric acid, activation temperature, activation time and heating rate.The optimum activation temperature is $500^{\circ} \mathrm{C}$, activation time is $3 \mathrm{~h}$, the impregnation ratio is $1: 2$.

\section{References}

[1] Dimitrios Kalderis, Sophia Bethanis, Panagiota Paraskeva,Production of activated carbon from bagasse and rice husk by a single-stage chemical activation method at low retention times[J],Bioresource Technology,2008,99:6809-6816

[2] K.Y. Foo, B.H. Hameed,Utilization of rice husks as a feedstock for preparation of activated carbon by microwave induced $\mathrm{KOH}$ and $\mathrm{K} 2 \mathrm{CO} 3$ activation[J],Bioresource Technology,2011,102: 9814-9817

[3] Kairan Zhu, Hao Fu, Jinghui Zhang,et al,Studies on removal of NH4+-N from aqueous solution by using the activated carbons derived from rice husk[J],biomass and bioenergy,2012,43:18-25

[4] Long Lin,Shang-Ru Zhai,Zuo-Yi Xiao et al,Dye adsorption of mesoporous activated carbons produced from $\mathrm{NaOH}$-pretreated rice husks[J],Bioresource Technology,2013,136:437-443

[5] Gao pin,Liu Zhenhong,Xue Gang,et al.Adsorption of phenol by Activated Carbon prepared from Waste Rice Straw by Chemical Activation with (NH4)2HPO4[J].Journal of Donghua University(Eng).2011, 28(6):552-558.

[6] Deng X H, Yue Y H, Gao Z. New Carbon-silica Composite Adsorbents from Elutrilithe [J]. Catalysis Today,1993,17:41-42

[7] Ramanu J R, Purusho T M, Chia M H. Processing and Characterization of Activated Carbon Coated Magnetic Particles for Biomedical Applications[J]. Materials Science and Engineering, 2007, 27(4): 659-664. 
[8] Ai K T. Activation of olive-seed waste residue using CO2 In a fluidized-bed Reactor[J]. Fuel Pross Technol, 1998,57(1):55-64

[9] Bo Guo,Liping Chang, Kechang Xie.Adsorption of Carbon Dioxide on Activated Carbon[J].Journal of Natural Gas Chemistry. 2006,15:223-229.

[10]SM Yakout.Adsorption kinetics modeling of benzene from aqueous solution onto rice husk activated carbon prepared by $\mathrm{H} 3 \mathrm{PO} 4$ activation[J].Journal- Indian Chemical Society, 2014, 91(10):1915-1924 